\title{
A Clustering Adaptive Neural Network Approach for Leaf Disease Identification
}

\author{
Neetu Chahal \\ Student, M.Tech \\ Deptt. Of Computer Sc., \\ ITM University \\ Gurgaon, Haryana
}

\author{
Anuradha \\ Asstt. Professor, \\ Deptt. Of Computer Sc, \\ ITM University, \\ Gurgaon, Haryana
}

\begin{abstract}
Agricultural image processing is having its significance to classify the agricultural objects as well as to identify the disease. These diseases are specific to the agricultural object or generic in nature. In this paper, an effective way is defined to identify the leaf disease over the plants. The work is here defined based on moment based analysis defined under clustering improved neural network approach. The work is here improved as a layered clustered and classification approach. In first stage, the identification of disease ROI is done. This identification is done using the CMeans Clustering Approach. Once the disease ROI is identified, the next work is to perform neural network approach for disease identification. The obtained results shows the effective recognition of disease area over the leaf images.
\end{abstract}

\section{Keywords}

Leaf Disease, ROI, Neural Network

\section{INTRODUCTION}

Agricultural image processing are having the significance on agricultural data and images to identify the objects and the disease over the available dataset. The work is here defined to recognize the image features and based on the prior analysis, the identification of object and the object disease is performed. This kind of identification is generally specialized to the auricular object as well as the part of the object. The disease areas include the fruits, leaf, flower based identification. Some of the diseases are generic and identified on any kind of images. In this work, a generic disease identification process is defined for leaf images. The work is here defined in two main stages. In first stage the disease ROI is identified and later on the comparison between the healthy part and disease part is done based on moment based analysis. The disease identification model for this work is given here under.

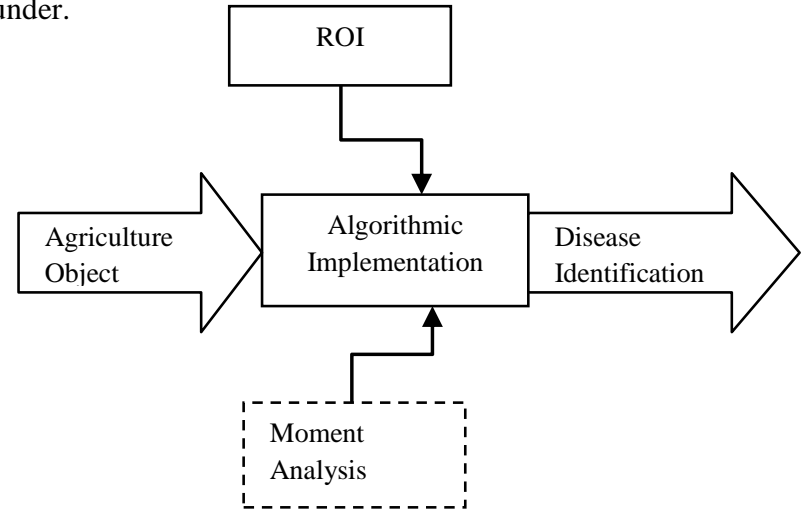

Fig 1: Disease Identification Model
Here figure 1 is showing the basic process of disease identification over the leaf images. As shown in the figure, the agricultural object is applied under two main algorithmic stags called ROI identification and moment analysis approach. The ROI identification will obtain the disease area over the image and the moment based analysis will separate the disease area and the normal good area over the image. Here dotted stage is dependent stage that will be applied once the object disease area will be identified. Over the image. The ROI extraction stage is here represented as

ROI=Extraction(InputFilteredImage,ColorModel, MorphOp)

Here the equation shows the extraction of effective image area based on the disease identification over the image so that the effective disease identification will be done over the image.

In this paper, an effective disease identification approach is presented based on mathematical operators and moment based analysis. The work is presented as two stage model. First stage is defined to extract disease ROI and second stage is defined to extract the disease area over the image. In this section the standard model for disease processing is defined. In section 2, the work defined by earlier researchers is presented. In section 3 , the proposed research methodology is defined. In section 4 , the results obtained from work is presented. In section 5, the conclusion obtained from work is shown.

\section{RELATED WORK}

Zhou Guoying[1] defined a work on the identification on Antagonistic Actinomyceters disease in soil. This work is defined to identify the problem analyze the soil components to identify the fungus disease. This model is defined to capture the fungus activity analysis so identify the abnormal feature quality so that the disease prediction will be performed under atmospheric conditions and culture conditions with bacterial stain.

Lixin $\operatorname{Li}[2]$ defined a work to provide the study on the bacterial impact on various crops and plants with atmospheric condition analysis. Author analyze the excess sludge with wastewater treatment so that the morphological cell properties with colony based optimization will be obtained. Author defined the analysis based on the various chemical vectors and relative component mixture to identify the relative problem. Haiguang Wang[3] has defined a work to detect the plant disease in images using neural network approach. Author defined a work to perform the disease recognition on plants and provided the formulation under the disease impact analysis so that the early tracking of the plant disease based on the plant image feature analysis will be obtained. Author applied the work on grape downy features to identify the leaf and rust problems. Author defined the color and shape based 
feature extraction so that the disease identification will be performed in an effective way. The feature extraction can be here performed under feature segment derivation. Author defined the PCA improved neural network model to perform disease identification and classification. Author also applied different classification model to analyze the predictive results.

Haiguang Wang[4] presented a hybrid mechanism to identify the plant disease based on leaf feature analysis. Author considered the various shape and color adaptive features for wheat plants. Author identify the rust, stripe and leaf based disease feature based disease identification and prediction. Author presented the shape and color feature adaptive model to perform the recognition at the early stage. Author defined the predictive model to perform the early detection of disease. Author used the PCA as the feature and distance adaptation approach and used the neural network as the recognition model to perform disease classification.

Nurul Hidayah Tuhid[5] has defined a predictive statistical approach for orchid disease analysis under color modeling. Author applied work on RGB features to identify the black leaf spots and identified as the orchid plant disease. Author defined a color segmentation approach to detect the critical image area using ROI extraction approach and later on applied the predictive classifier to perform the disease detection and classification. Haiguang Wang[6] has defined an effective recognition approach using back propagation network. Author defined a work for automatic diagnosis of plant diseases and improves the plant disease under disease type. These disease types includes grape downy and grape powdery. Author extract the object features and generated the pattern measures to perform the classification. After image preprocessing including image compression, image cropping and image denoising, K_means clustering.

Sanjeev S Sannakki[7] has presented a leaf disease detection and classification using neural network. The goal of proposed work is to diagnose the disease using image processing and artificial intelligence techniques on images of grape plant leaf. In the proposed system, grape leaf image with complex background is taken as input. Thresholding is deployed to mask green pixels and image is processed to remove noise using anisotropic diffusion. Then grape leaf disease segmentation is done using K-means clustering. Asma Akhtar[8] has presented an automated way for disease detection and analysis. Author implied the intelligent learning model to generate the leaf feature patterns at the early stage and later on generate the feature segment based on the region level classification model. Author analyzes the signal under feature adaption. The classification model is here applied to perform the recognition and classification. Author applied the work on different data values to perform the classification and recognition under disease type and the leaf type. The recognition is here performed using SVM classifier and the classification class is obtained from the work.

Lung-Jen Wang[9] defined a hybrid image enhancement and the classification approach. Author defined a non linear approach to perform the image enhancement for the blurred images and the estimate the image cost based on which the classification of the image is done. Author defined a multi parametric pre-processing so that the image features will be acquired correctly. Author defined a series of scaling and clipping approaches so that the the effective image filtration will be done. Once the image enhancement is done, at the next stage the classification is done under the opportunistic cost evaluation approach.
Another work on the image classification was done by Tianquian Peng in year 2003 on remote sensing images. Author defined the work using the neural network based approach. Author defined the information analysis approach under the evidence theory analysis as well asthe neural network approach. The proposed work is also divided in two main stages, first to perform the image filtration so that the better classification results will be obtained. Later on back propagation classification along with evidence theory is implemented to perform the image classification[10]. Another work on the image feature analysis approach for the image classification was presented by S.L. Wang in year 2007. Author defined a histogram analysis approach to perform the content based feature analysis. Author defined the feature identification and the color information extraction approach to perform the effective classification. Author defined the resolution analysis based quantization approach so that the neighboring pixel analysis and feature extraction approach will be introduced and adopt so that the effective feature evaluation will be performed. Author defined the feature representation approach under different real time aspects. Author defined a hybrid model using the adaboost and SVM approach so that effective classification will be done. The obtained results shows that the work has improve the effectiveness of the classification[11].

\section{PROPOSED WORK}

The presented work is here defined to identify the plant disease by using the hybrid approach. The work is here represented as the layered model. In this layered model, the first stage is to improve the image features. To perform this image feature improvement histogram analysis is applied in this work. Once the normalized image is obtained, the next work is to apply the segmentation to identify the effective disease region over the image. To perform this effective region identification, fuzzy CMeans algorithm is implemented in this work.

The Clustering approach has separated the key area over the image and the segmented effective ROI is obtained. The clustering model perform the intensity based division over the image. This division has separated the foreground and the background area. Later on the separation is performed in terms of effective good and bad area. This bad area over the image is identified as the ROI. Finally, this weighted region is trained using neural network model to identify the disease probability in the image. This weighted neural network approach identified the disease over the image. The presented work is here defined as the work model. This work model is given in figure 2 .

In this work a cluster adaptive distance learning method is adopted to perform the image partitioning. The Euclidian distance is here considered as the major distance measure to divide the mage in sub segments and similarity criteria based recognition is performed. The adaptive hierarchical method is here applied to divide the image in feature segments so that the relative effective clusters will be identified. The clustering model is here based on the weight modeling and updated in each round to optimize the centroid. The algorithmic approach adapted for cluster formation are given here under 


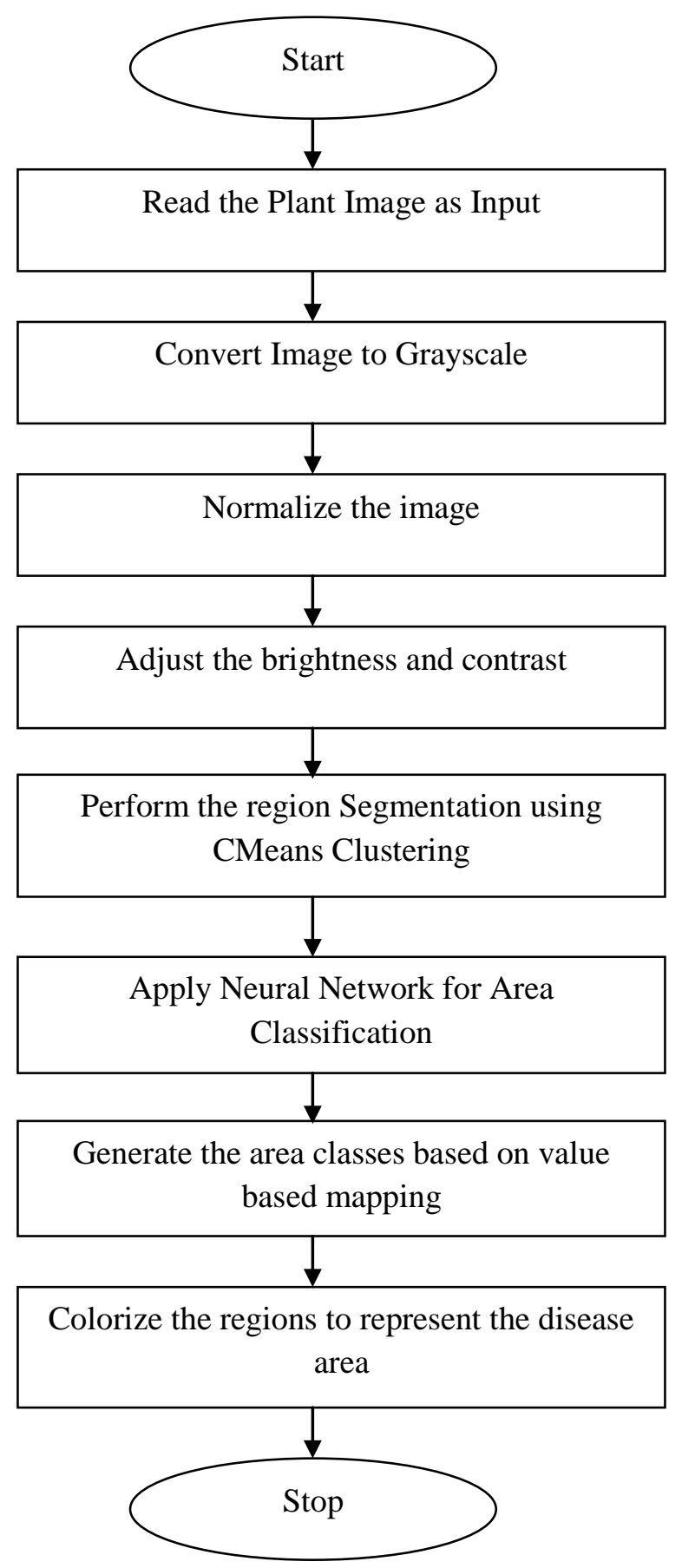

Fig 2: Proposed Model

The steps for a standard k-means clustering algorithm are as follows.

1. Accept the image intensity point in the form of matrix as the input vector for clusters.

2. Specify $\mathrm{N}$ number of clusters to perform the area division or segmentation

3. Perform the nearest neighbor mapping under distance based analysis to identify the cluster members.

4. Shift the centroid by analyzing to the mean value.
5. Repeat from step 3 till the movement over the centers is not stopped

Once the high level clustering is performed, the neural network model is applied to identify the disease over the image. The experimentation related to this work is given in next section.

\section{RESULTS}

The presented work is here defined to identify the plant disease based on clustering improved neural network model. To perform the experimentation, the random images are collected from external web sources. These images are real time images available in different resolution. The results obtained from the work are shown for a sample image.

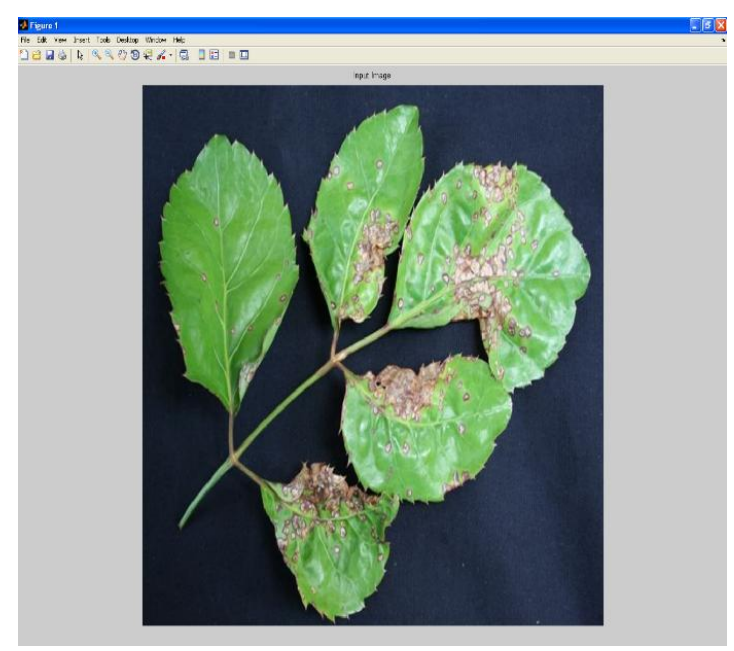

Fig 3: Input Image

Here figure 3 is showing the input image on which proposed algorithm is applied. The image is a color image present in jpg format. The figure is showing the input image having the disease in it. The two level segmentation is here applied to identify the disease. The first level segmentation is here performed using CMeans clustering approach and neural network model is applied to perform the recognition. The disease detected image is shown in figure 4.

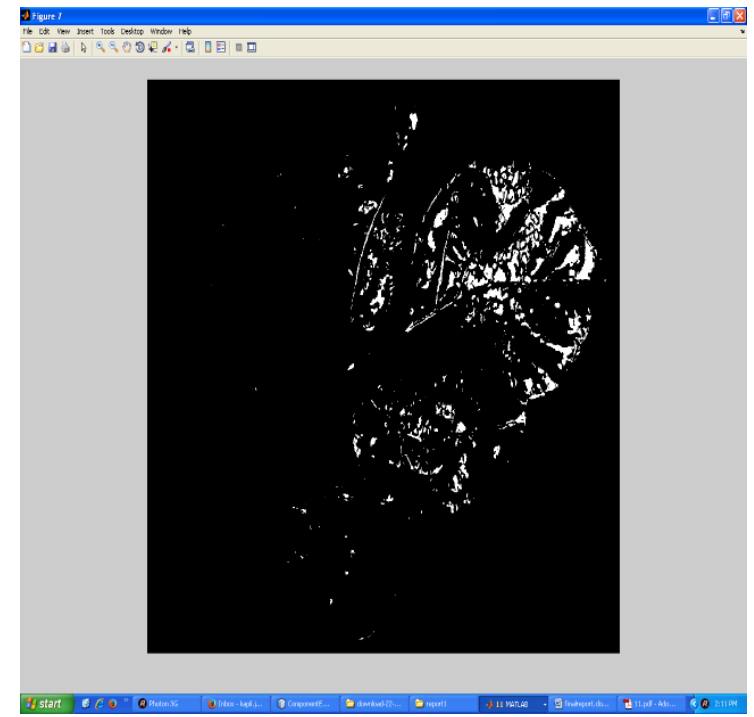

Fig 4 : Segmentation Result 
Here figure 4 is showing the segmenation results. The region ROI is here identified on which the neural network is applied to identify the disease over the image.

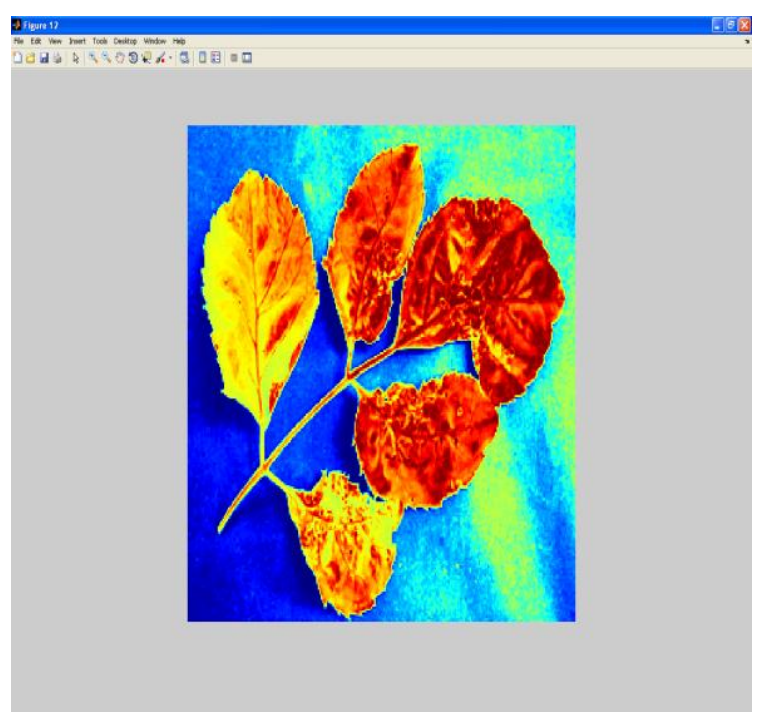

Fig 5 : Disease Detected Image

Here figure 5 is showing the colorized disease detected image. Here the blue area is showing the external area that is not the part of main object itself. The red aea is the good image area and the brown area is the actual disease area identified using proposed algorithmic approach. The result shows the effective identification of disease over the image.

\section{CONCLUSION}

In this paper, a neural network based adaptive model is presented to identify the leaf disease. The work is here defined for generic disease. In first stage of this model, effective disease region is identified using fuzzy CMeans approach. Later on neural network model is applied to identify the disease accurately.

\section{REFERENCES}

[1] Zhou Guoying,2010 " Fungistatic activity and identification of Antagonistic Actinomycetes to camellia diseases from Soil",International Conference on
Challenges in Environmental Science and Computer Engineering Volume I, 475-478.

[2] Lixin Li," Isolation, Identification and optimization of Culture Conditions of Photosynthetic Bacteria", 2011, ICBBE, 1-4.

[3] Haiguang Wang," Application of Neural Networks to Image Recognition of Plant Diseases", 2012 International Conference on Systems and Informatics (ICSAI 2012) 2159-2164.

[4] Haiguang Wang," Image Recognition of Plant Diseases Based on Principal Component Analysis and Neural Networks", 2012 8th International Conference on Natural Computation (ICNC 2012) 246-251

[5] Nurul Hidayah Tuhid," A Statistical Approach for Orchid Disease Identification using RGB Color", 2012 IEEE Control and System Graduate Research Colloquium (ICSGRC 2012) 382-385

[6] Haiguang Wang," Image Recognition of Plant Diseases Based on Backpropagation Networks", 2012 5th International Congress on Image and Signal Processing (CISP 2012) 894-900

[7] Sanjeev S Sannakki, "Diagnosis and Classification of Grape Leaf Diseases using Neural Networks", 4th ICCCNT 2013

[8] Asma Akhtar," Automated Plant Disease Analysis (APDA): Performance Comparison of Machine Learning Techniques",2013 11th International Conference on Frontiers of Information Technology

[9] Lung-Jen Wang," Combined Opportunity Cost and Image Classification for Non-Linear Image Enhancement", 2012 Sixth International Conference on Complex, Intelligent, and Software Intensive Systems 135-140

[10] Tianqiang Peng," A Remote Sensing Image Classification Method Based on Evidence Theory and Neural Networks", IEEE Int. Conf. Neural Networks \&Signal Processing Vol I, pp. 240-244.

[11] S.L.Wang," Information-Based Color Feature Representation For Image Classification", ICIP 2007 Vol 6, pp. VI-353-VI-356 